\title{
Characterization of Anamnestic T-cell Responses Induced by Conventional Vaccines against Contagious Bovine Pleuropneumonia
}

\author{
Philippe Totte ${ }^{1,2 *}$, Aboubakar Yaya ${ }^{3}$, Amadou Sery ${ }^{4}$, Hezron Wesonga ${ }^{5}$, Abel Wade ${ }^{3}$, Jan Naessens ${ }^{6}$, \\ Mamadou Niang ${ }^{4}$, François Thiaucourt ${ }^{1,2}$
}

1 Centre International de Recherche en Agronomie pour le Développement, UMR CMAEE, Montpellier, France, 2 Institut National de Recherche Agronomique, UMR1309 CMAEE, Montpellier, France, $\mathbf{3}$ Laboratoire National Vétérinaire, Garoua, Cameroon, $\mathbf{4}$ Laboratoire Central Vétérinaire, Bamako, Mali, $\mathbf{5}$ Kenya Agricultural Research Institute, Kikuyu, Kenya, 6 International Livestock Research Institute, Nairobi, Kenya

\begin{abstract}
A better understanding of how $\mathrm{T} 1$ vaccination confers immunity would facilitate the rational design of improved vaccines against contagious bovine pleuropneumonia (CBPP). We show here that mycoplasmas-induced recall proliferation and IFN- $\gamma$ responses are detected in cattle that received multiple shots of $\mathrm{T} 1$ vaccines. These anamnestic responses were under the strict control of $\mathrm{CD}^{+} \mathrm{T}$ lymphocytes. Moreover, CD62L expression indicated that both $\mathrm{CD}^{+}$effector memory (Tem) and central memory $(\mathrm{Tcm}) \mathrm{T}$ lymphocytes are elicited in these animals. Comparative analysis with data from cattle that completely recovered from CBPP infection revealed similar anamnestic T-cell responses albeit at a lower magnitude for T1vaccinated animals, particularly in the Tcm compartment. In conclusion, we discuss how our current understanding of T-cell responses will contribute to ongoing efforts for the improvement of future CBPP vaccines.
\end{abstract}

Citation: Totte P, Yaya A, Sery A, Wesonga H, Wade A, et al. (2013) Characterization of Anamnestic T-cell Responses Induced by Conventional Vaccines against Contagious Bovine Pleuropneumonia. PLoS ONE 8(2): e57509. doi:10.1371/journal.pone.0057509

Editor: Odir A. Dellagostin, Federal University of Pelotas, Brazil

Received January 7, 2013; Accepted January 23, 2013; Published February 28, 2013

Copyright: (C) 2013 Totte et al. This is an open-access article distributed under the terms of the Creative Commons Attribution License, which permits unrestricted use, distribution, and reproduction in any medium, provided the original author and source are credited.

Funding: This study was funded in parts by grants from the European Union, under the supervision of OAU/IBAR (Kenya), and from the Australian aid program. J.N. was sponsored by a CSIRO-AUSaid grant. The funders had no role in study design, data collection and analysis, decision to publish, or preparation of the manuscript.

Competing Interests: The authors have declared that no competing interests exist.

*E-mail: philippe.totte@cirad.fr

\section{Introduction}

Improved vaccines are needed to control the spread of contagious bovine pleuropneumonia (CBPP), a devastating respiratory disease of cattle caused by Mycoplasma mycoides subsp. mycoides biotype Small Colony $(\mathrm{MmmSC})$, and a substantial economic burden for sub-Sahara Africa $[1,2,3]$. Indeed, the only vaccines currently in use are live vaccines, derived from the $\mathrm{T} 1$ strain and empirically attenuated through serial passage in embryonated eggs before production in artificial growth media [4]. Unfortunately these vaccines possess variable efficacy, induce short-lived immunity necessitating costly annual booster immunizations, and still retain some virulence causing sometimes adverse reactions at inoculation sites [5,6]. Although some technical advances were accomplished in quality control and storage, recent efforts to address the limited efficacy of CBPP vaccines by increasing vaccine dosage and formulation resulted in little success $[2,7]$.

Comprehensive information on the nature of immunity induced by $\mathrm{T} 1$ vaccines will help improve their efficacy. Also, it may lead to the definition of predictors of vaccine efficacy that will allow a simple and rapid screening approach of novel vaccine formulations before fastidious and costly clinical trials in ruminants. As far as T1 vaccines are concerned, and apart from one report by Roberts et al in 1973 [8], research on immune responses triggered by vaccination has focused exclusively on humoral immunity. These studies indicate that antibody responses elicited by $\mathrm{T} 1$ vaccines are generally low and short-lived, and, thus, not appropriate to assess the immune status of animals or to serve as predictors of vaccine success [5,9]. Preliminary work on cell-mediated immunity (CMI) failed to detect sensitization of lymphocytes in vaccinated animals but the methods used were of low sensitivity and specificity [8].

Here, we have analyzed T-cell immune responses induced by $\mathrm{T} 1$ vaccines using highly sensitive methods such as enzyme-linked immunospot (ELISPOT) for the detection of $\mathrm{MmmSC}$-specific IFN- $\gamma$ production, and carboxyfluorescein diacetate succinimidyl ester (CFSE) to measure lymphocytes proliferation. The two currently approved vaccines, namely, T1/44 and its streptomycinresistant derivate, $\mathrm{T} 1 \mathrm{sr}$, were used in this study. In addition, single immunization was compared to multiple annual booster immunization known to improve overall herd immunity. Also, we investigated the induction of both effector memory (Tem) and central memory $(\mathrm{Tcm}) \mathrm{T}$ cells given their importance in maintaining long-term protective immunity [10]. Finally, a comparative analysis was performed between data from the present study and our knowledge of CMI induced in animals that recovered from CBPP. 


\section{Materials and Methods}

\section{Ethics Statement}

The African research institutions involved in the animal experiments did not have ethic committees operational at the time of the study. However, each received authorization to conduct the study from their respective ministry/government department namely the Ministry of livestock Development in Kenya, the Ministry of Livestock and Fisheries in Mali and the Ministry of Livestock, Fisheries and Animal Husbandry in Cameroon. In addition, all experiments were carried out according to the guidelines in the guide to the Care and Use of Experimental Animals provided by the French Ministry of Agriculture.

\section{Animals and Vaccinations}

Naïve zebu cattle above 3 years of age were purchased from CBPP-free areas in each country and were confirmed negative for antibodies to $M m m S C$ by ELISA. Animals were dewormed and treated against ticks when needed. They were confined in a paddock or a brick house, where they were fed on hay, water and mineral supplements. Vaccinations were performed as follow: i) at KARI in Kenya: animals received a single subcutaneous injection of a T1/44 vaccine batch; ii) at CVL in Mali, animals received two intramuscular injections at one month interval of a T1-SR vaccine batch; iii) at LANAVET in Cameroon, animals previously vaccinated twice on a yearly basis were purchased from a region were annual CBPP vaccination takes place under LANAVET's supervision. At LANAVET, these animals received a third subcutaneous injection of a T1/44 vaccine batch. All vaccines preparations were quality controlled by the pan African veterinary vaccine centre (PANVAG) in Ethiopia and used at a dosage of $10^{7}$ viable mycoplasmas per injection [2]. Control nonvaccinated animals received PBS only and were kept with the vaccinated animals.

\section{Proliferation Assays and Flow Cytometry}

PBMC were isolated from peripheral blood as described previously [11] one month after the last vaccination and stored at $-80^{\circ} \mathrm{C}$ in cryotubes containing $2-5 \times 10^{7}$ cells $/ \mathrm{ml}$ in fetal calf serum supplemented with $10 \%$ DMSO until shipment to CIRAD under liquid nitrogen. Upon thawing, PBMC were loaded with CFSE (Invitrogen, France) at a final concentration of $1 \mu \mathrm{M}$ before incubation for 9 days with $5 \mu \mathrm{g} / \mathrm{ml}$ of heat-inactivated $\mathrm{MmmSC}$ (strain $\mathrm{T} 1 / 44$ ) or $2.5 \mu \mathrm{g} / \mathrm{ml}$ of the mitogen Concanavalin A (ConA) as a positive control and as described before [12]. Phenotype analysis of PBMC was performed as described previously with minor modifications [11,12]. Briefly, cells were harvested and surface stained with the following primary monoclonal antibodies (mAb): mAb IL-All (IgG2a) for CD4, mAb BAQ92A (IgG1) for CD62L, mAb GC6A (IgM) for CD45R and mAb GC42Al (IgG1) for CD45RO (all obtained from VMRD, Pullman, WA). Cells were then washed and stained with a cocktail of fluorochrome-conjugated, isotype-specific antibodies (Tebu, France). Debris and dead cells were excluded from analysis based on size and inclusion of 7-amino-actinomycin D (7-AAD) as a viability dye. Four-color analyses were performed with a FACScanto flow cytometer equipped with the FACSDiva TM software (BD Biosciences) after the acquisition of at least 5,000 events. Control isotype antibodies were used to evaluate nonspecific binding and to set gates and quadrants delineating positive populations. Proliferation levels are expressed as percentages of CFSElow cells which correspond to decreased CFSE fluorescence intensities relative to that in undivided cells. They measure the cumulated proliferation since day 0 irrespective of the number of division cycles.

\section{IFN- $\gamma$ Assays}

The Bovigam ${ }^{\text {TM }}$ ELISA (Prionics AG, Switzerland) was used to assess IFN- $\gamma$ production in 5-days old supernatants of PBMC cultured as above. Briefly, supernatants were collected after centrifugation and stored at $-20^{\circ} \mathrm{C}$ until assayed according to the manufacturer's instructions; results are expressed as mean O.D. values of duplicates.

The bovine IFN- $\gamma$ ELISpot ${ }^{\text {plus }}$ kit (Mabtech, Sweden) was used to establish the numbers of ex vivo antigen-responding cells in cultured PBMC and following the manufacturer's instructions. Briefly, the assay was carried out in duplicates in polyvinylidene fluoride (PVDF) 96-well plates coated with a capture mAb specific for bovine IFN- $\gamma$. PBMC $\left(2 \times 10^{5} /\right.$ well) resuspended in RPMI1640 supplemented with 10\% FCS (Eurobio), 2 mM glutamine, antibiotics and 2-mercaptoethanol (Sigma) were stimulated with the same antigens as in the proliferation assay and incubated for $20 \mathrm{~h}$ at $37^{\circ} \mathrm{C}$ in a humidified atmosphere with $5 \% \mathrm{CO}_{2}$. After extensive washing, the plates were incubated with a detection $\mathrm{mAb}$ against bovine IFN- $\gamma$. Finally, IFN- $\gamma$ secreting cells or spots were stained using a combination of alkaline phosphatase (ALP), 5Bromo-4-chloro-3-indolyl phosphate (BCIP) and nitroblue tetrazolium (NBT). Spot forming cells (SFG) were enumerated using an automated ELISPOT reader (Biosys, Germany). Results are presented as the mean number of $M m m S C$-specific IFN- $\gamma$-spot forming cells $/ 10^{6}$ PBMC.

\section{Depletion Studies}

Cells were depleted of $\mathrm{CD} 4^{+} \mathrm{T}$ cells by positive selection using a magnetic activated cell sorting system (Macs, Miltenyi Biotec, France) as previously described (12). Briefly, cells were stained with mAb ILA11 (IgG2a) against bovine CD4 (VMRD, USA) and incubated with Macs microbeads conjugated to goat anti-mouse IgG $(\mathrm{H}+\mathrm{L})$ prior to separation on MS columns and according to the manufacturer's instructions. Unbound cell fractions collected from the columns repeatedly contained less than $5 \% \mathrm{CD}^{+} \mathrm{T}$ cells as confirmed by flow cytometry.

\section{Statistics}

A nonparametric Mann-Whitney U-test (http://elegans.som. vcu.edu/leon/stats/) was used to analyze differences between vaccinated and nonvaccinated animals. A difference was considered to be significant at a $P$ value of $<0.05$.

\section{Results}

\section{Anamnestic Cellular Responses are Detected in Cattle that Received Multiple Shots of T1 Vaccines}

At individual levels, MmmSC-specific recall proliferation in vaccinated cattle could only be detected in animals that received three shots of T1 vaccines (Fig. 1). However, at group levels, these vaccinated cattle could not be discriminated from nonvaccinated animals when recall proliferation of total PBMC was assessed (Fig. la). It is only when proliferation among $\mathrm{CD} 4^{+} \mathrm{T}$ lymphocytes was measured that significantly $(P=0.03)$ higher responses were detected in the vaccinated group versus the nonvaccinated group (Fig. 1b).

MmmSC-induced recall IFN- $\gamma$ responses in vaccinated animals were also detected at substantial levels only in animals receiving multiple vaccine injections (Fig. 2). At group levels, both ELISA $(P=0.028)$ and ELISPOT $(P=0.016)$ methods allowed discrimination between vaccinated and nonvaccinated animals (Fig. 2a\&b). 

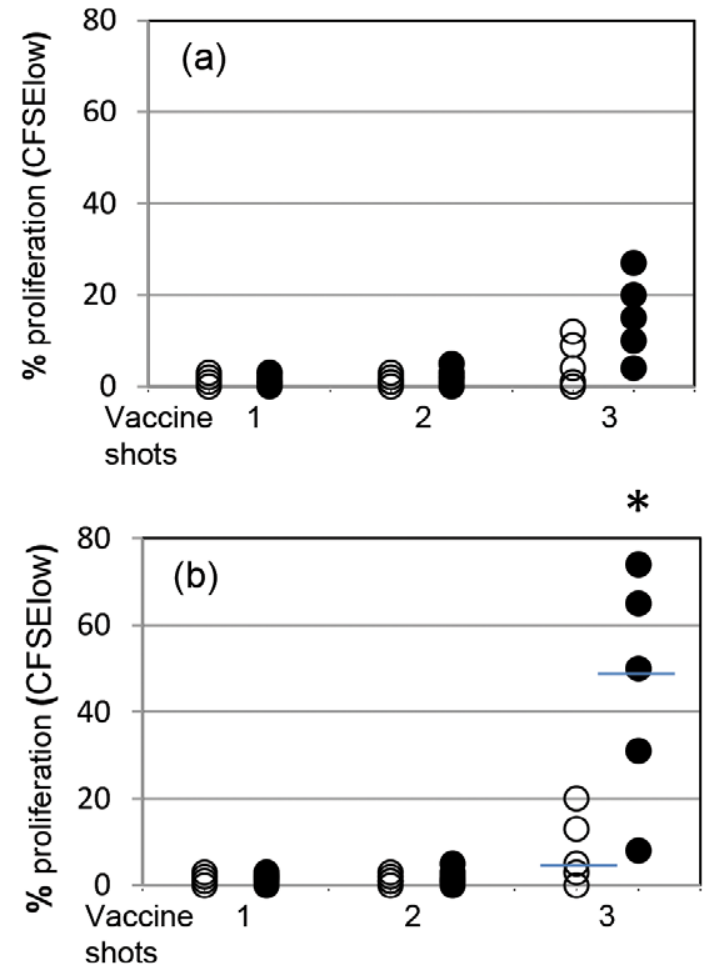

Figure 1. MmmSC-induced recall proliferative responses of pbmc (a), and $\mathrm{CD4}^{+} \mathrm{T}$ cells (b), collected from nonvaccinated (open circles) and vaccinated (closed circles) animals $(n=5)$ one month after single, double and triple vaccine inoculations. Results represent the net effect of $\mathrm{MmmSC}$ stimulation (i.e., stimulated cultures minus non-stimulated cultures) and are representative of 2 independent experiments. Bars indicate median values and asterisks represent significant difference between vaccinated and naïve groups. doi:10.1371/journal.pone.0057509.g001

Recall proliferative and IFN- $\gamma$ responses to the mitogen ConA were comparable for all groups irrespective of the vaccination status and number of vaccine shots (see Fig. S1).

\section{Anamnestic Cellular Responses Induced by T1 Vaccines are under the Strict Control of $\mathrm{CD}^{+}{ }^{+} \mathrm{T}$ cells}

In vaccinated animals showing the strongest $\mathrm{T}$-cell responses, MmmSC-induced recall proliferation was evident among cells that did not belong to the CD4 sub-type although to a lesser extent than in the $\mathrm{CD} 4^{+}$population. Given the potential inhibitory properties of $\mathrm{CD}^{+} \mathrm{T}$ lymphocytes (i.e., Tregulatory or Treg) it was of interest to analyse immune responses occuring in their absence. Therefore, depletion studies were performed and clearly indicated that both proliferation and IFN- $\gamma$ production induced by MmmSC Ags required the presence of $\mathrm{CD}^{+} \mathrm{T}$ lymphocytes (Fig. 3).

\section{Both $\mathrm{CD}^{+} \mathrm{Tem}$ and $\mathrm{Tcm}$ are Elicited by $\mathrm{T} 1$ Vaccines}

We have shown previously that expression of CD62L among proliferating $\mathrm{CD}^{+} \mathrm{T}$ lymphocytes could discriminate between Tem and Tcm in MmmSC presensitized animals [13]. Here, we have assessed the contribution of both populations in anamnestic $\mathrm{T}$-cell responses of vaccinated cattle, focusing on animals that responded above nonvaccinated animals (i.e., 4 out of 5), to concentrate on vaccine-induced $\mathrm{T}$-cell responses. We found that viable $\mathrm{CD} 4^{+} \mathrm{T}$ lymphocytes actively proliferating in response to MmmSC comprised both $\mathrm{CD}_{6} 2 \mathrm{~L}^{+}$and $\mathrm{CD} 62 \mathrm{~L}^{-}$populations
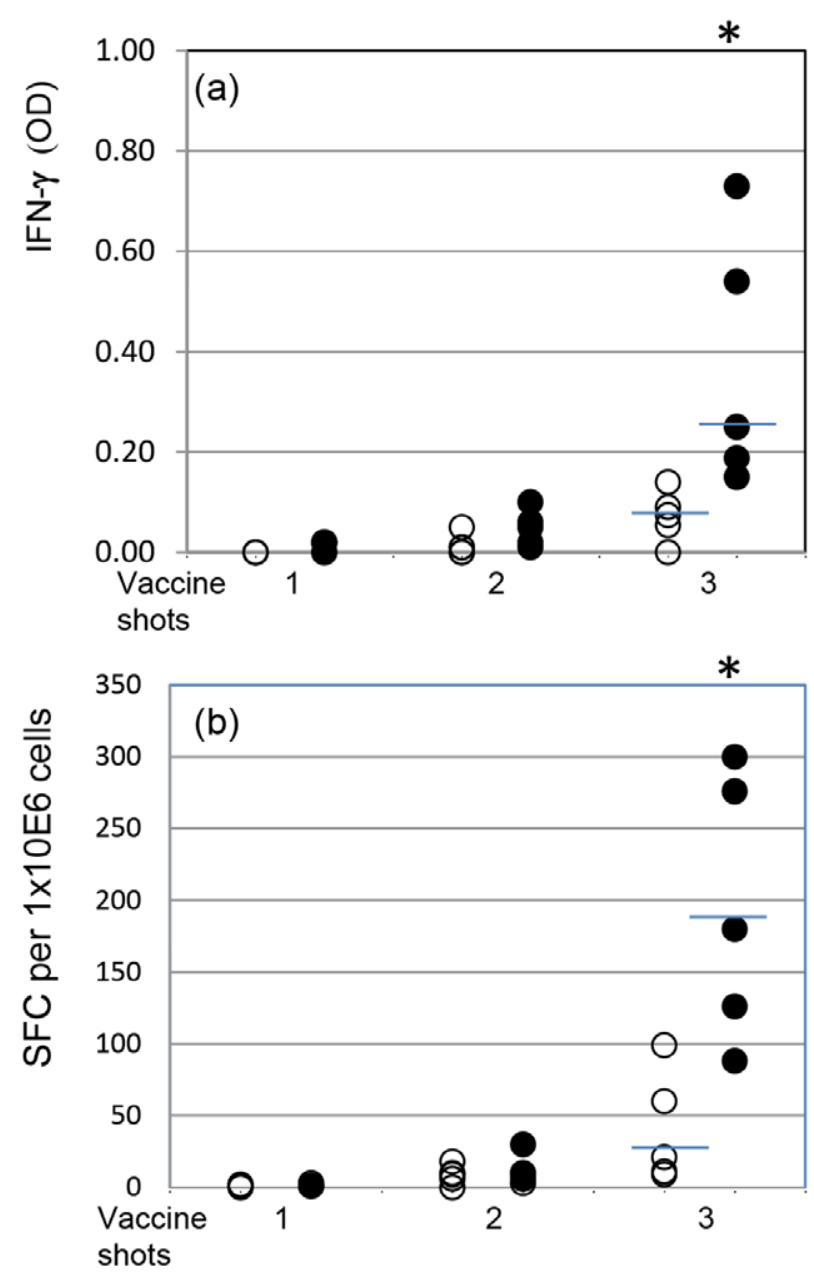

Figure 2. MmmSC-induced recall IFN- $\gamma$ responses measured by ELISA (a) and ELISPOT (b) using pbmc collected from nonvaccinated (open circles) and vaccinated (closed circles) animals $(n=5)$ one month after single, double and triple vaccine inoculations. Results represent the net effect of $M m m S C$ (i.e., stimulated cultures minus non-stimulated cultures) and are representative of two experiments. Bars indicate median values and asterisks represent significant difference between vaccinated and naïve groups. doi:10.1371/journal.pone.0057509.g002

(Fig. 4). In general, the percentage of $\mathrm{CD} 2 \mathrm{~L}^{+}$cells slightly exceeded the percentage of $\mathrm{CD}^{-} \mathrm{L}^{-}$cells among proliferating $\mathrm{CD}^{+} \mathrm{T}$ lymphocytes (Table 1) whereas in non stimulated CD4 the ratio was $45 \pm 5 \%$.

Analysis of activation/memory markers indicated strong upregulation of $\mathrm{CD} 45 \mathrm{RO}$ and down regulation of CD45R surface expression by proliferating $\mathrm{CD} 4^{+} \mathrm{T}$ lymphocytes (Fig. 5). It can be observed from Fig. 5 that, among proliferating CD4, the majority of $\mathrm{CD}_{62} \mathrm{~L}^{-}$and $\mathrm{CD} 62 \mathrm{~L}^{+}$also followed that same expression pattern (i.e., upregulation of CD45RO and downregulation of CD45R) which is indicative of a memory phenoytpe.

\section{Discussion}

The paucity of data on the immunogenicity of $\mathrm{T} 1$ vaccines against CBPP, and more specifically on CMI, has prompted this study. Our goal is to improve our understanding of protective immunity and facilitate the design of more effective CBPP vaccines that are urgently needed. Three African countries took part in the study to account for differences in national veterinary 
(a)

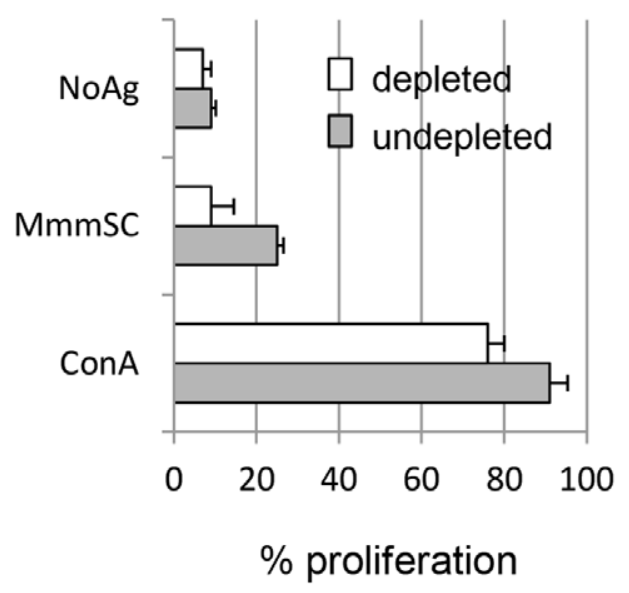

(b)

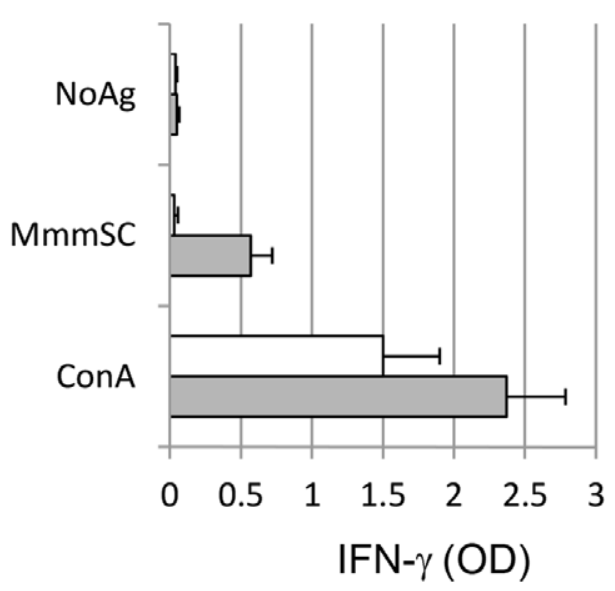

Figure 3. Effect of CD4 depletion on MmmSC-induced recall proliferation (a) and IFN- $\gamma$ (b) responses of pbmc collected from vaccinated animals. Cells were incubated in the absence (NoAg) or presence of $M m m S C$ Ags (MmmSC), or in the presence of the mitogen Concanavalin $\mathrm{A}(\mathrm{ConA})$ as a positive control. Results are expressed as mean percentages ( \pm standard deviation) from three animals. doi:10.1371/journal.pone.0057509.g003

specificities and practices. This also allowed comparison of different vaccine strains and vaccination regimens.

In the first place, recall proliferation and IFN- $\gamma$ production by PBMC, two markers of CMI, were chosen to assess the presence of anamnestic T-cell responses in vaccinated animals. Significant $M m m S C$-induced recall proliferation and IFN- $\gamma$ responses, both at individual and group levels, were detected exclusively in cattle that received three shots of $\mathrm{T} 1$ vaccines. These results confirm pioneer studies by Roberts and colleagues indicating that no recall activation of lymphocytes could be detected in cattle vaccinated two months previously in the tail tip with a single dose of the T1 vaccine [8]. A booster immunization one month later had little effect as shown in the present study. A possible explanation is that live $\mathrm{T} 1$ vaccines induce apoptosis in lymphocytes as has been shown in vitro for the T1/44 strain [14]. In our study, there was no obvious signs left of apoptosis in PBMC as indicated by uncompromised responses to the mitogen ConA (see Figure S1). Additional longitudinal studies are needed to fully explore the possibility that transient CMI is induced by a single shot of $\mathrm{T} 1$ vaccines.

Further characterization of CMI elicited by multiple shots of T1 vaccines underlined the determinant role of $\mathrm{CD} 4^{+} \mathrm{T}$ lymphocytes in recall $M m m S C$-induced proliferation and IFN- $\gamma$ responses.
Although the contribution of other T-cell sub-types cannot be excluded, $\mathrm{MmmSC}$-induced responses in vitro were under the strict control of $\mathrm{CD}^{+} \mathrm{T}$ lymphocytes as shown by depletion studies. Moreover, $\mathrm{CD} 4^{+} \mathrm{T}$-cell responders elicited by multiple injections of $\mathrm{T} 1$ vaccines comprised both Tem- and Tcm-like sub-types as suggested by CD62L surface expression. Interestingly, a predominant role for $\mathrm{CD}^{+}{ }^{+} \mathrm{T}$ lymphocytes in CMI, including both Tem and Tcm, has also been reported for cattle that fully recovered from CBPP [13]. Full recovery from clinical disease is known to induce life-long protective immunity against CBPP $[15,16]$. However, although qualitatively comparable, the magnitude of CMI was lower in T1-vaccinated cattle versus recovered cattle as indicated by several parameters (Table 1 and ref 13): 1) 2/ $5 \mathrm{v} / \mathrm{s} 10 / 10$ animals had more than $50 \%$ of CFSElow cells among $\mathrm{CD}^{+} \mathrm{T}$ cells after in vitro stimulation; 2) $2 / 5 \mathrm{v} / \mathrm{s} 10 / 10$ animals showed an increase in total $\mathrm{CD}^{+} \mathrm{T}$ cells among stimulated PBMC; 3) 0/5 v/s 10/10 animals showed above 70\% CD62L ${ }^{+}$ cells among proliferating $\mathrm{CD} 4^{+} \mathrm{T}$ cells which is a marker for $\mathrm{Tcm}$ in our model [13]. This is consistent with the short-lived immunity afforded by $\mathrm{T} 1$ vaccines observed in the field which justifies costly annual booster immunizations campaigns [16].

Although not a final proof, this study further supports the idea that both $\mathrm{CD}^{+} \mathrm{Tem}$ and $\mathrm{Tcm}$ play a role in protective immunity

Table 1. Effect of in vitro MmmSC stimulation on $\mathrm{CD}^{+} \mathrm{T}$ lymphocytes collected from cbpp-vaccinated cattle.

\begin{tabular}{|c|c|c|c|c|c|}
\hline \multirow[t]{2}{*}{$\begin{array}{l}\text { Animal } \\
\text { number }\end{array}$} & \multicolumn{2}{|c|}{$\begin{array}{l}\% \mathrm{CD4}^{+} \mathrm{T} \text { cells in } \\
\text { cultures stimulated }^{\mathrm{a}} \text { with: }\end{array}$} & \multicolumn{2}{|c|}{$\%$ proliferation ${ }^{\mathbf{b}}$ among $\mathrm{CD4}^{+} \mathrm{T}$ cells } & \multirow[t]{2}{*}{$\begin{array}{l}\% \mathrm{CD}^{2} 2 \mathrm{~L}^{+} \text {cells among proliferating } \\
\mathrm{CD4}^{+} \mathrm{T} \text { cells }\end{array}$} \\
\hline & NoAg & MmmSC & NoAg & MmmSC & \\
\hline 1 & $21.5 \pm 3$ & $34 \pm 4$ & $7 \pm 2$ & $60 \pm 14$ & $62 \pm 4$ \\
\hline 2 & $24.5 \pm 5$ & $23.5 \pm 7$ & $4 \pm 3$ & $37 \pm 12$ & $60 \pm 2$ \\
\hline 3 & $15 \pm 2$ & $18 \pm 1$ & $6 \pm 2$ & $25 \pm 6$ & $51 \pm 1$ \\
\hline 4 & $20 \pm 5$ & $40 \pm 4$ & $3 \pm 2$ & $51 \pm 14$ & $61 \pm 5$ \\
\hline
\end{tabular}

apbmc were loaded with CFSE and incubated for 9 days with inactivated MmmSC before cell surface staining and analysis by flow cytometry as described in material\&methods. Results are expressed as mean ( $+/-\mathrm{SD})$ percentages of 2 experiments.

${ }^{b}$ Percentages of CFSElow cells within the gated $\mathrm{CD} 4{ }^{+}$population.

doi:10.1371/journal.pone.0057509.t001 
(a)

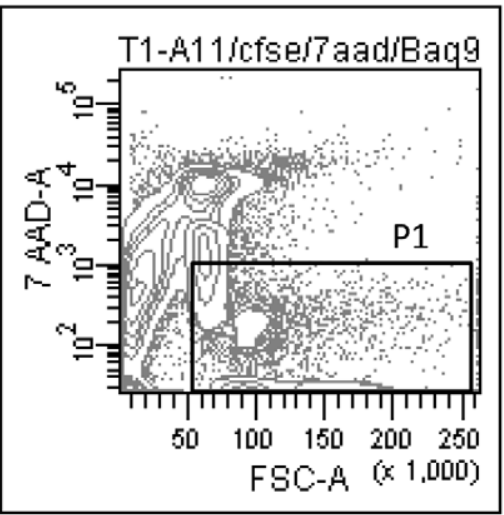

(b)

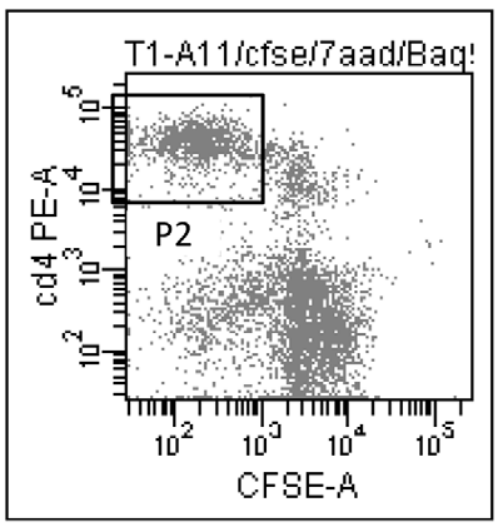

(c)

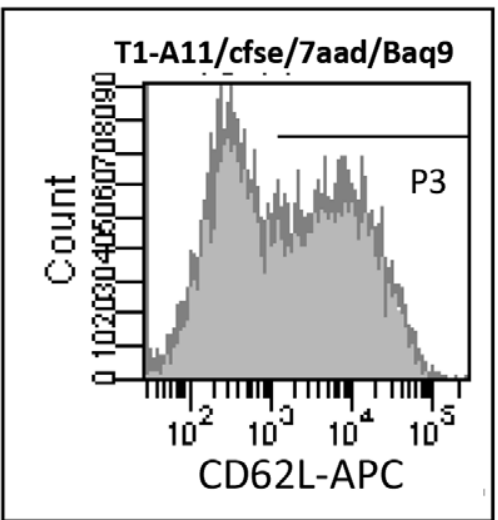

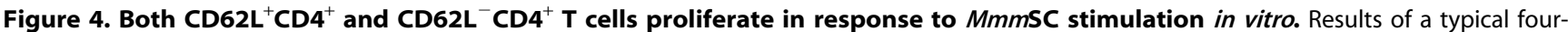
color flow cytometric analysis are shown for one vaccinated animal. Cells were loaded with CFSE and incubated for 9 days with inactivated MmmSC before cell surface staining and analysis by flow cytometry as follows: (a) a first gate (P1) was used to exclude dead cells (7AAD+) and cell debris $(\mathrm{FSC}<50)$; (b) a second gate (P2) was set, among P1 gated cells, to delineate viable and proliferating CD4 ${ }^{+} \mathrm{T}$ cells $\left(\mathrm{CFSElow}\right.$ or CFSE $\left.<1 \times 10^{3}\right)$; and $(\mathrm{c})$, histograms were opened on gate $\mathrm{P} 2$ to obtain the percentage of $\mathrm{CD} 62 \mathrm{~L}^{+}$cells among proliferating $\mathrm{CD} 4^{+} \mathrm{T}$ cells (i.e., $\mathrm{P} 3$ ).

doi:10.1371/journal.pone.0057509.g004

since booster immunizations are associated with increased protection rate of $\mathrm{T} 1$ vaccines [17]. There is however a need for studies on CMI taking place in vaccinated/recovered cattle undergoing CBPP challenge to confirm the role of $\mathrm{CD}^{+} \mathrm{T}$ cells in protection. Moreover, the contribution of functionally different $\mathrm{CD}^{+}{ }^{+}$effectors or Tem (i.e., Th1, Th2, Th17, Treg, polyfunctional CD4) will have to be clarified to understand the mechanisms underlying protective immunity [18]. This is especially true since severe CBPP may be associated with excessive cytokine production [19] and, thus, inappropriate immunization may exacerbate disease rather than protect. Additionally, this will allow identifi- cation of suitable correlates of protection that would facilitate future vaccine evaluation in cattle. Another important aspect of vaccine efficacy is long-term immunity particularly for CBPP to avoid expensive annual booster immunizations. Tcm mediate long-term immunity and have been associated with protection against chronic diseases [10, 20, 21, and 22], Moreover, in bovine tuberculosis, $\mathrm{T} \mathrm{cm}$ responses are detected long after Tem responses have waned and they are good correlates of vaccine-induced protection [23,24].

Altogether, these results suggest that the immunogenicity of T1 vaccines is poor but that it can be increased by several booster (a)

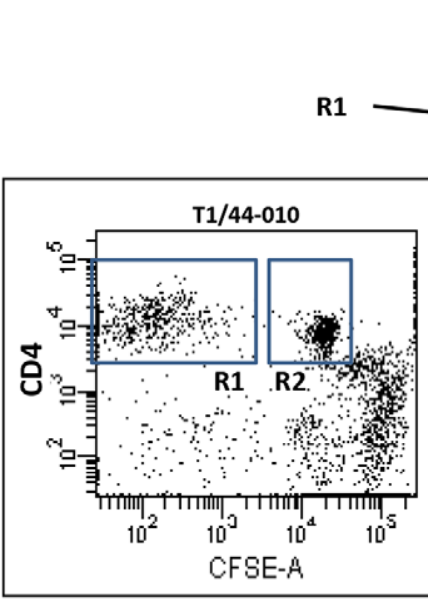

R2

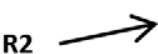

(b)

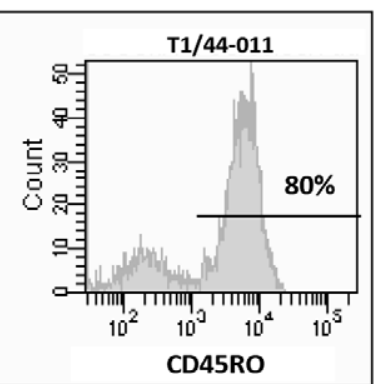

(c)
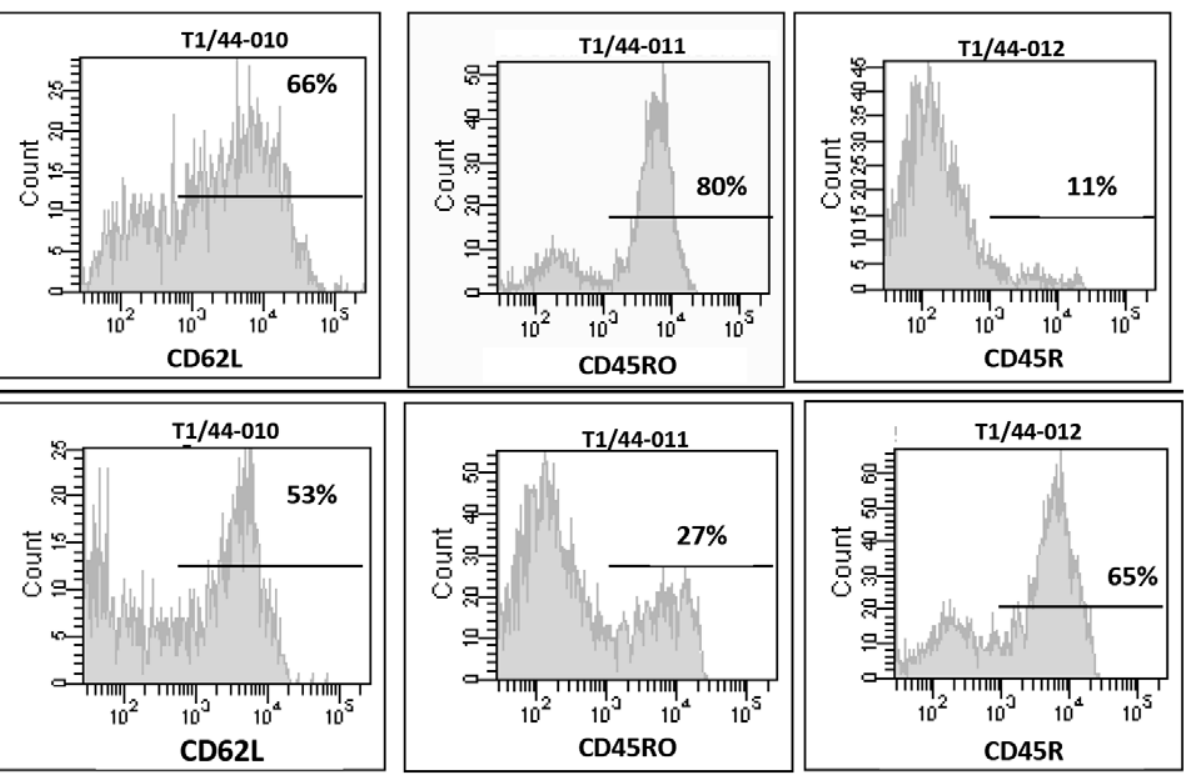

Figure 5. Cell surface expression of CD62L (a), and CD45RO (b), and CD45R (c) markers by proliferating (R1 gate) and nonproliferationg (R2 gate) CD4 ${ }^{+}$T cells stimulated with MmmSC Ags. Percentages of positive cells within gated populations are shown on histograms.

doi:10.1371/journal.pone.0057509.g005 
immunizations. In that case, animals developed CMI that was qualitatively comparable to animals that have fully recovered from CBPP infections. However, quantitative analysis suggests that there is room for substantial increase in CMI, particularly in the Tcm compartment, even after three shots of $\mathrm{T} 1$ vaccines. This warrants further work to assess new boosting strategies other than homologous booster immunization. Our results also further support the idea that protection against CBPP is somehow associated with $\mathrm{CD} 4^{+} \mathrm{Tem}$, the precise phenotype of which remains to be elucidated, as well as $\mathrm{CD}^{+} \mathrm{Tcm}$. Therefore, markers of these responses have potential as predictors of vaccine efficacy and deserve further evaluation in future CBPP challenge studies.

\section{Supporting Information}

Figure S1 Recall responses to the mitogen ConA measured by: i) proliferation among $\mathrm{CD} 4^{+} \mathrm{T}$ lymphocytes (a), ii) IFN- $\gamma$ production measured by ELISA (b) and ELISPOT (c). Cells were collected

\section{References}

1. Tambo NE, Maina WO, Ndi C (2006) An estimation of the economic impact of contagious bovine pleuropneumonia in Africa. Rev Sci Tech Oie 3: 999-1011.

2. Thiaucourt F, Aboubakar Y, Wesonga H, Manso-Silvan L, Blanchard A (2004) Contagious bovine pleuropneumonia vaccines and control strategies: recent data. In: Control of Infectious Animal Diseases by Vaccination, A. Schudel and M. Lombard, Editors, Karger:Basel. p. 99-111.

3. (2006) In:4th FAO-OIE-AU/IBAR-IAEA. Consultative Group Meeting on CBPP in Africa. Rome: FAO.

4. Sheriff D, Piercy SE (1952) Experiments with an avianised strain of the organism of contagious bovine pleuropneumonia. Vet Rec 64: 615-621.

5. Thiaucourt F, Yaya A, Wesonga H, Hubschle OJ, Tulasne JJ, et al. (2000) Contagious bovine pleuropneumonia. A reassessment of the efficacy of vaccines used in Africa. Ann. N Y Acad Sci 916: 71-80.

6. Revell SG (1973) Local reactions following contagious bovine pleuropneumonia vaccination in Zambia, Trop Anim Health Prod 5: 246-252.

7. Nkando I, Ndinda J, Kuria J, Naessens J, Mbithi F, et al. (2012) Efficacy of two vaccine formulations against contagious bovine pleuropneumonia (CBPP) in Kenyan indigenous cattle. Res Vet Sci 2: 568.

8. Roberts DH, Windsor RS, Masiga WN, Kariavu CG (1973) Cell-mediated immune response in cattle to Mycoplasma mycoides var. mycoides, Infect Immun 8: 349-354.

9. Hamsten C, Neiman M, Schwenk JM, Hamsten M, March JB, et al. (2009) Recombinant surface proteomics as a tool to analyze humoral immune responses in bovines infected by Mycoplasma mycoides subsp. mycoides small colony type. Mol Cell Proteomics 11: 2544-54

10. Zaph C, Uzonna J, Beverley SM, Scott P (2004) Central memory T cells mediate long-term immunity to Leishmania major in the absence of persistent parasites. Nat Med 10: 1104

11. Dedieu L, Balcer-Rodrigues V, Yaya A, Hamadou B, Cisse O, et al. (2005) Gamma interferon-producing CD4 T-cells correlate with resistance to Mycoplasma mycoides subsp. Mycoides S.C. infection in cattle. Vet Immunol Immunopathol 107: 217-233.

12. Totté P, Rodrigues V, Yaya A, Hamadou B, Cisse O, et al. (2008) Analysis of cellular responses to Mycoplasma mycoides subsp. mycoides small colony biotype associated with control of contagious bovine pleuropneumonia. Vet Res 39: $1-11$. from nonvaccinated (open circles) and vaccinated (closed circles) animals $(\mathrm{n}=5)$ one month after single, double and triple vaccine inoculations. Results represent the net effect of ConA (i.e., stimulated cultures minus non-stimulated cultures).

(TIF)

\section{Acknowledgments}

We express our appreciation to the staff of national veterinary institutes for excellent technical help and their dedication to the welfare of test animals. We are very grateful to V. Rodrigues for technical help with flow cytometry.

\section{Author Contributions}

Reviewed critically the manuscript: AY MN JN AW FT. Conceived and designed the experiments: PT AY HW AS MN FT. Performed the experiments: PT AS AW HB JN. Analyzed the data: PT FT. Wrote the paper: PT.

13. Totté P, Duperray G, Dedieu L (2010) CD62L defines a subset of pathogenspecific bovine CD4 with central memory cell characteristics. Dev Comp Immunol 34: 177-82.

14. Dedieu L, Chapey E, Balcer-Rodrigues V (2005) Mycoplasma mycoides ssp. mycoides biotype small colony-secreted components induce apoptotic cell death in bovine leucocytes. Scand J Immunol 6: 528-38.

15. Hudson JR (1968) Contagious bovine pleuropneumonia. The keeping properties of the V5 vaccine used in Austtralia. Aust Vet J 44: 123-9.

16. Masiga WN, Domenech J, Windsor RS (1996) Manifestation and epidemiology of contagious bovine pleuropneumonia in Africa. Rev Sci Tech 18: 59-65.

17. Wesonga HO, Thiaucourt F (2000) Experimental Studies on the Efficacy of T1sr and T1/44 Vaccine Strains of Mycoplasma mycoides Subspecies mycoides (Small Colony) against a Field Isolate Causing Contagious Bovine Pleuropneumonia in Kenya - Effect of a Revaccination. Rev Elev Med Vet Pays Trop 4: 313-318

18. Seder RA, Darrah PA, Roederer M (2008) T-cell quality in memory and protection: implications for vaccine design. Nat Rev Immunol 8: 247-258.

19. Sacchini F, Luciani M, Salini R, Scacchia M, Pini A (2012) Plasma levels of TNF- $\alpha$, IFN- $\gamma$, IL- 4 and IL-10 during a course of experimental contagious bovine pleuropneumonia. BMC Vet Res 8: 44

20. Keating SM, Bejon P, Berthoud T, Vuola JM, Todryk S, et al. (2005) Durable human memory $\mathrm{T}$ cells quantifiable by cultured enzyme-linked immunospot assays are induced by heterologous prime boost immunization and correlate with protection against malaria. J Immunol 175: 5675-80.

21. Soloff AC, Liu X, Gao W, Day RD, Gambotto A, et al. (2009) Adenovirus 5and 35-based immunotherapy enhances the strength but not breadth or quality of immunity during chronic SIV infection. Eur J Immunol 9: 2437-49.

22. Goletti D, Butera O, Bizzoni F, Casetti R, Girardi E, et al. (2006) Region of difference 1 antigen-specific CD4+ memory $\mathrm{T}$ cells correlate with a favorable outcome of tuberculosis. J Infect Dis 7: 984-92.

23. Whelan AO, Wright DC, Chambers MA, Singh M, Hewinson RG, et al. (2008) Evidence for enhanced central memory priming by live Mycobacterium bovis BCG vaccine in comparison with killed BCG formulations. Vaccine 2: 166-73.

24. Hope JC, Thom ML, McAulay M, Mead E, Vordermeier HM, et al. (2011) Identification of surrogates and correlates of protection in protective immunity against Mycobacterium bovis infection induced in neonatal calves by vaccination with M. bovis BCG Pasteur and M. bovis BCG Danish. Clin Vaccine Immunol 3: 373-9. 